\title{
233 Evidence that post-contrast delayed enhancement identified by cardiovascular magnetic resonance is an arrhythmogenic substrate in hypertrophic cardiomyopathy
}

\author{
A Selcuk Adabag1, Barry J Maron², Evan Appelbaum³, Caitlin J Harrigan³, \\ Jacqueline L Buros ${ }^{3}$, C Michael Gibson ${ }^{4}$, John R Lesser², \\ Constance A Hanna ${ }^{2}$, James E Udelson ${ }^{4}$, Warren J Manning ${ }^{3}$ and \\ Martin S Maron*4
}

Address: ${ }^{1}$ VA Medical Center, Minneapolis, MN, USA, ${ }^{2}$ Minneapolis Heart Institute Foundation, Minneapolis, MN, USA, ${ }^{3}$ Beth Isreal Deaconess Medical Center; Perfuse Core Laboratory and Data Coordinating Center, Boston, MA, USA and ${ }^{4}$ Tufts-New England Medical Center, Boston, MA, USA

* Corresponding author

from I Ith Annual SCMR Scientific Sessions

Los Angeles, CA, USA. I-3 February 2008

Published: 22 October 2008

Journal of Cardiovascular Magnetic Resonance 2008, I0(SuppI I):A94 doi:I0.I I86/I532-429X-I0-SI-A94

This abstract is available from: http://jcmr-online.com/content/I0/SI/A94

(c) 2008 Adabag et al; licensee BioMed Central Ltd.

\section{Introduction}

Delayed enhancement (DE) on contrast-enhanced cardiovascular magnetic resonance (CMR), representative of myocardial fibrosis is relatively common in the left ventricle (LV) of patients with hypertrophic cardiomyopathy (HCM). However, the clinical significance and arrhythmogenic potential of this finding remains unresolved.

\section{Purpose}

Therefore, the objective of the present study was to assess the prevalence and frequency of spontaneous tachyarrhythmias in HCM with regard to DE on contrastenhanced CMR.

\section{Methods}

A total of 177 HCM patients (age $41 \pm 16$ years; 95\% asymptomatic or mildly symptomatic) were evaluated by 24-hour ambulatory Holter ECG and contrast-enhanced CMR.

\section{Results}

A total of 72 patients (41\%) had DE (transmural in 39/ 72 ), occupying $8.5 \pm 7.8$ percent of the LV wall. Presence of premature ventricular contractions (PVCs), couplets and nonsustained ventricular tachycardia (NSVT) were more common in patients with DE than those without DE (PVCs: $89 \%$ vs. $72 \%$; couplets: $40 \%$ vs. $17 \%$; NSVT: $28 \%$ vs. $4 \% ; \mathrm{p}<0.0001-0.007)$. Patients with DE also had greater numbers of PVCs $(202 \pm 655$ vs. $116 \pm 435)$, couplets $(1.9 \pm 5$ vs. $1.2 \pm 10)$, and NSVT runs $(0.4 \pm 0.8 v$ s. $0.06 \pm 0.4)$ than did non-DE patients (all $\mathrm{p}<0.0001)$; DE was an independent predictor of NSVT (relative risk 7.3, $95 \%$ CI $2.6-20.4 ; \mathrm{p}<0.0001)$. Of note, however, extent (\%) of DE was similar in patients with and without PVCs ( $8.2 \%$ vs. $9.1 \% ; \mathrm{p}=0.93)$, couplets $(8.5 \%$ vs. $8.4 \% ; \mathrm{p}=$ $0.99)$ and NSVT $(8.3 \%$ vs. $8.5 \% ; \mathrm{p}=0.35)$.

\section{Conclusion}

In this large HCM cohort with no or only mild symptoms, myocardial fibrosis detected by CMR was associated with greater likelihood and increased frequency of ventricular tachyarrhythmias (including NSVT) on ambulatory Holter ECG. Therefore, contrast-enhanced CMR identifies HCM patients having increased susceptibility to ventricular tachyarrhythmias with implications for sudden death risk stratification. 